\title{
An extension of max autoregressive models*
}

\author{
Philippe NaVeau ${ }^{\dagger}$, ZhenguUn Zhang ${ }^{\ddagger}, \S$ AND Bin Zhu
}

To model clustered maxima behaviors in time series analysis, max-autoregressive (MAR) and moving maxima (MM) processes are naturally adapted from linear autoregressive (AR) and moving average (MA) models. Yet, applications of MAR and MM processes are still sparse due to some difficulties of parameter inference and some abnormality of the processes. Basically, some ratios of observations can take constant values in MAR models. The objective of this present work is to introduce a new model that is closely related to the MAR processes and is free of the aforementioned abnormality. A logarithm transformation of the new model leads to time series models with log-positive alpha stable noises and hidden max Gumbel shocks. Theoretical properties of the new models are derived.

AMS 2000 subject Classifications: Primary 60G70, 62M10; secondary $62 \mathrm{G} 32$.

Keywords AND PHRASEs: Extreme Value Theory, Dependence, Gumbel distribution, Autoregressive model.

\section{MOTIVATION}

Recordings of daily, weekly or yearly maxima in environmental time series are classically fitted by the Generalized Extreme Value (GEV) distribution that originates from the well established Extreme Value Theory (EVT). To capture temporal dependencies and clustered peak values, linear autoregressive (AR) processes and moving average (MA) processes offer a simple and elegant framework. [3] introduced Max-ARMA processes; [11] generalized Deheuvels' [4] moving minima processes to multivariate maxima of moving maxima (M4) processes. However, applications of the MaxARMA processes and the M4 processes have been rare due to some inferential difficulties and some abnormality of the processes. Ratios of consecutive observations drawn from either a Max-ARMA process or an M4 process can take constant values. These constant ratios form signature patterns in simulated processes, see $[3,16]$ for example. These signature patterns have not been observed in real data analysis

\footnotetext{
*The authors are grateful to two referees for their valuable comments ${ }^{\dagger}$ Part of this work has been supported by the EU-FP7 ACQWA Project (www.acqwa.ch) under Contract Nr 212250, by the PEPERGIS project (ADEME), by the ANR-MOPERA project, by the ANRMcSim project and by the MIRACCLE-GICC project.

$\ddagger$ Corresponding author.

$\S$ Zhang's research was supported by NSF Grant DMS-0804575.
}

even if processes are transformed into Fréchet scales as required in either a Max-ARMA model or an M4 model. As a result, models with more flexible dependence patterns are needed. This paper aims to propose such a kind of models.

One of the simple Max-ARMA processes is the following one

$$
Y_{t}=\max \left(a Y_{t-1}, \epsilon_{t}\right)
$$

where $a<1$ is a positive constant and $\epsilon_{t}$ corresponds to an iid sequence of unit Fréchet random variables, i.e. $P\left(\epsilon_{t}<\right.$ $u)=\exp (-1 / u), u>0$. We note that the marginal choice of $\epsilon_{t}$ being unit Fréchet is not essential. Suppose that $\epsilon_{t}$ has the heavy-tailed extreme value distribution $\exp \left\{-1 / u^{\tau}\right\}$ for some $\tau>0$, then one can transform back to the unit Fréchet case by taking $1 / \tau$ powers in the Max-ARMA recursion (1); see [3] for detailed arguments.

This simple model can be viewed as an entry point of our discussions and it can also be rewritten as $Y_{t}=$ $\max _{j=0,1, \ldots}\left(a^{j} \epsilon_{t-j}\right)$. Its main drawback is that the random ratio $Y_{t} / Y_{t-1}$ equals the constant $a$ whenever we have $a Y_{t-1}>\epsilon_{t}$. This implies that the value of $a$ can be estimated exactly. In practice it is highly improbable to find an application with such a behavior. To remove this undesirable effect, we propose the following model

$$
Z_{t, \alpha}=c \max \left(a\left(S_{t, \alpha} Z_{t-1, \alpha}\right)^{\alpha}, \epsilon_{t}\right),
$$

where $c \in(0,1]$ is a scale parameter, $a \in(0,1]$ is an autoregressive parameter, and the sequence $\left\{S_{t, \alpha}\right\}$ is independent of the iid sequence $\left\{\epsilon_{t}\right\} .\left\{S_{t, \alpha}\right\}$ represents an iid sequence of positive $\alpha$-stable variables defined by its Laplace transform

$$
E(\exp (-u S))=\exp \left(-u^{\alpha}\right), \text { for all } u \geq 0,
$$

where $\alpha \in(0,1]$. Here $S_{t, \alpha}$ and $Z_{t-1, \alpha}$ in (2) are also independent.

Recall that a random variable $S$ is said to be stable if for all non-negative real numbers $c_{1}, c_{2}$, there exists a positive real $a$ and a real $b$ such that $c_{1} S_{1}+c_{2} S_{2}$ is equal in distribution to $a S+b$ where $S_{1}, S_{2}$ are iid copies of $S$. The case $\alpha=1$ in (2) means that $S=1$. This situation corresponds to model (1). Whenever $\alpha<1$, the ratio $Z_{t, \alpha} / Z_{t-1, \alpha}$ depends on the random variable $S_{t, \alpha}$, and consequently it cannot be equal to a constant. As a result, model (2) does not possess the aforementioned limitation of model (1). 

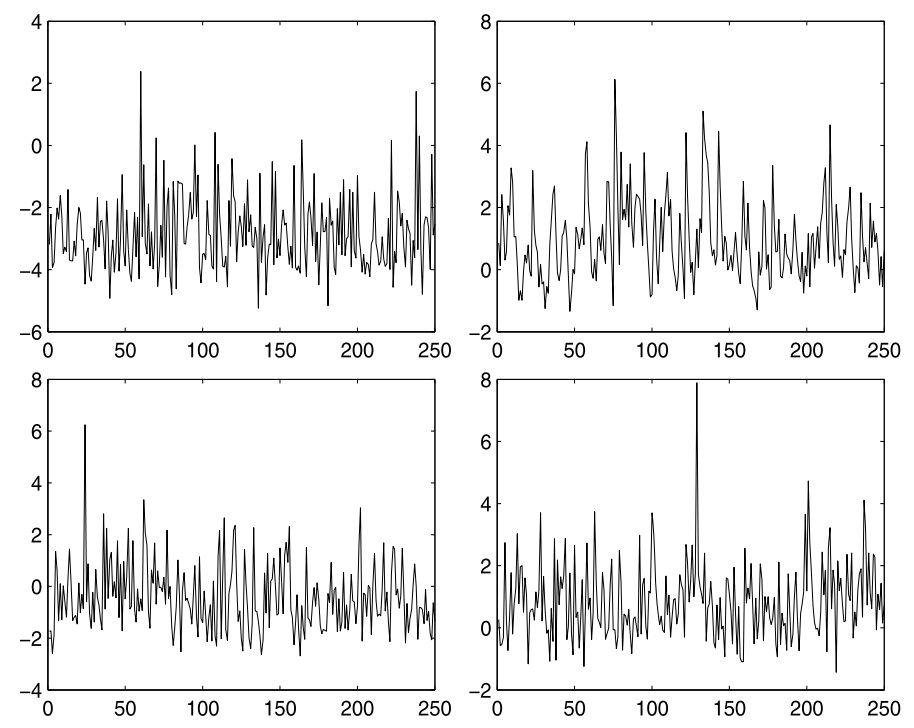

Figure 1. 250 Observations in model (4) with different sets of parameter values of $(a, c, \alpha)$. Respectively, the top-left uses $(0.3047,0.0260,0.3488)$, the top-right uses $(0.7330,0.5100$, $0.6630)$, the bottom-left uses $(0.5758,0.2365,0.1601)$, and the bottom-right uses $(0.7208,0.8124,0.2137)$.

One building block behind (2) is an additive relationship between Gumbel and $\log$ positive $\alpha$-stable variables. If $X$ is Gumbel distributed with parameters $\mu$ and $\sigma$ and is independent of $S$, a positive $\alpha$-stable variable, then the sum $X+\sigma \log S$ is also Gumbel distributed with parameters $\mu$ and $\sigma / \alpha$. Such an additive property has been recently studied by [15] in an environmental context and [5] in a mixture context. In the literature, $[2,7,13]$ also worked with such distributions in survival analysis and the modeling of multivariate extremes.

Our proposed model (2) contains three parameters. First, $\alpha$ is a shape parameter. When either $Z_{t-1, \alpha}$ or $S_{t, \alpha}$ is very large, $\left(S_{t, \alpha} Z_{t-1, \alpha}\right)^{\alpha}$ has a tendency to become smaller comparing with the original values. Second, $a$ is the parameter that describes the auto-regressive of our model. Third, the parameter $c$ can be viewed as a scale parameter or a location parameter after a $\log$ transformation of $Z_{t, \alpha}$. A logarithm transformation of (2) yields the following time series model:

$$
X_{t, \alpha}=\mu+\max \left\{\gamma+\alpha \log \left(S_{t, \alpha}\right)+\alpha X_{t-1, \alpha}, \xi_{t}\right\},
$$

where $\mu=\log (c), \quad \gamma=\log (a), \quad X_{t, \alpha}=\log \left(Z_{t, \alpha}\right), \quad \xi_{t}=$ $\log \left(\epsilon_{t}\right)$. In the model, $\mu$ is a location parameter, and both $X_{t, \alpha}$ and $\xi_{t}$ are Gumbel distributed. We can regard (4) as a time series model with log of positive $\alpha$ stable noises $\log \left(S_{t, \alpha}\right)$ and hidden max Gumbel shocks $\xi_{t}$. The idea is as follows. Suppose that $\xi_{t}=-\infty$ for all $t$. Then model (4) is a pure autoregressive signal process. Alternatively, suppose that $P\left(\xi_{t}>-\infty\right)=1$ in (4) at time $t$. If the signal value of $\xi_{t}$ is stronger than the signal resulted from the autoregressive signal process, then $\xi_{t}$ is the new observed signal value, i.e. the signal process is altered by a hidden $(\max )$ Gumbel shock. Figure 1 illustrates four different simulated processes using four different sets of parameter values in (4).

It is clear that model (4) generates skewed data and asymmetry in the upper and lower tails. Model (4) shares the spirit of the seminal nonlinear time series model, i.e. threshold autoregression models introduced by [14]. Model (4) uses a random threshold $\xi_{t}$ and takes value of the threshold itself as long as the value is larger than the computed autoregressive sum. This new model can also be regarded as a model with an infinite number of change points.

Model (2) and model (4) being basically equivalent, we will only consider (2) in the subsequent sections and derive its theoretical properties.

Before closing this section, we can remark that at least two extensions of model (2) are possible. The following general form

$$
Z_{t, \alpha}=c \max \left(\left[S_{t, \alpha} \max _{1 \leq i \leq p}\left\{a_{i} Z_{t-i, \alpha}\right\}\right]^{\alpha}, \epsilon_{t}\right)
$$

corresponds to a max autoregressive process with positive alpha stable moving coefficients and unit Fréchet shocks. We denote this process as $\operatorname{MAP}_{\alpha}(p)$. Another possible extension for model (2) follows the idea of [5] to take advantage of the $\alpha$-stability. This allows to introduce

$$
Z_{t, \alpha}=c \max \left(a\left\{\left[\sum_{j=0}^{\infty} c_{j} S_{t-j, \alpha}\right] Z_{t-1, \alpha}\right\}^{\alpha}, \epsilon_{t}\right) .
$$

The rest of the paper is structured as follows. In sections 2 and 3 , we present some theoretical properties of model (2), i.e. $\operatorname{MAP}_{\alpha}(1)$ processes. Section 3.2 deals with statistical estimations of parameters in $\mathrm{MAP}_{\alpha}(1)$ models with the positive $\alpha$ stable noises being Lévy distributed. Simulation examples are shown in Section 4. In Section 5, we fit a $\mathrm{MAP}_{\alpha}(1)$ model to weekly maxima of river flow rate data for two rivers: the Eagle River and the Crystal River, located in the mountains of western Colorado in the United States. Section 6 concludes. In Appendix, we present all technical derivations of theoretical results and proofs of propositions.

\section{THEORETICAL PROPERTIES OF $\operatorname{MAP}_{\alpha}(1)$ PROCESSES}

\subsection{Stationarity}

The following proposition shows that model (2) quickly produces a stationary process as $t$ increases.

Proposition 1. In the context of (2), suppose that the distribution of $Z_{0, \alpha}$ is Fréchet with scale parameter $b_{0}>0$, i.e. $P\left(Z_{0, \alpha} \leq z\right)=\exp \left(-b_{0} / z\right)$. Then $Z_{t, \alpha}$ is Fréchet with scale parameter satisfying

$$
b_{t}=a c b_{t-1}^{\alpha}+c
$$


for $t=1,2, \ldots$, and $\left\{b_{t}\right\}$ has a limit d satisfying

$$
a c d^{\alpha}+c=d
$$

as $t \rightarrow \infty$.

Furthermore, if $b_{0}=d$, we have for any constant values $z_{0}, z_{1}, \ldots$,

$$
\begin{aligned}
& P\left(Z_{0, \alpha} \leq z_{0}, \ldots, Z_{t, \alpha} \leq z_{t}\right) \\
& =P\left(Z_{k, \alpha} \leq z_{0}, \ldots, Z_{k+t, \alpha} \leq z_{t}\right), \\
& \quad \text { for any } t \geq 0 \text { and } k \geq 0
\end{aligned}
$$

which implies that the process is stationary.

We note that if $b_{0}=d$ satisfies $(7)$, the derivation in the proof of Proposition 1 indicates that model (2) corresponds to a stationary process in which the marginal distribution is Fréchet with the scale parameter being $d$.

On the other hand, if $b_{0}$ is not equal to $d$, then $b_{t}$ converges at least linearly, and it has a rate of approximately $(d-c) \alpha / d$. Numerically, except the case of $a=c=\alpha=1$, in a few iterations $(t<20), b_{t}$ has already closed to $d$ up to many decimal places. For this reason, we will assume the process generated from (2) is stationary with $d$ as its parameter, i.e. we assume $b_{0}=d$.

We note that in model (2), it is not necessary to require $a<1$. In the case of $a=1$, we still can have stationary processes, which will be seen in our real data analysis.

\subsection{Causality}

In Max-ARMA model, [3] called a stationary process causal if it can be expressed as a max-linear process. Following their lead, we call a stationary process causal if there exist constants $\beta_{j} \geq 0, j \geq 0$ and

$$
Z_{t, \alpha}=\beta_{0} \epsilon_{t} \vee\left[\bigvee_{j \geq 1} \beta_{j}\left(\prod_{i=0}^{j-1} S_{t-i}^{\alpha^{i+1}}\right) \epsilon_{t-j}^{\alpha^{j}}\right]<\infty \text { a.s. }
$$

To simplify notations, the subscript $\alpha$ was dropped in $S_{t, \alpha}$ that simply becomes $S_{t}$. We now study the causal properties of model (2). Substituting (8) in our proposed $\mathrm{MAP}_{\alpha}(1)$ process (2), we have

$$
\begin{aligned}
\beta_{0} \epsilon_{t} \vee \beta_{1} S_{t}^{\alpha} \epsilon_{t-1}^{\alpha} \vee\left[\bigvee_{j \geq 2} \beta_{j}\left(\prod_{i=0}^{j-1} S_{t-i}^{\alpha^{i+1}}\right) \epsilon_{t-j}^{\alpha^{j}}\right] \\
=c \epsilon_{t} \vee a c \beta_{0}^{\alpha} S_{t}^{\alpha} \epsilon_{t-1}^{\alpha} \vee\left[\bigvee_{j \geq 1} a c \beta_{j}^{\alpha} S_{t}^{\alpha}\left(\prod_{i=0}^{j-1} S_{t-1-i}^{\alpha^{i+2}}\right) \epsilon_{t-1-j}^{\alpha^{j+1}}\right] \\
=c \epsilon_{t} \vee a c \beta_{0}^{\alpha} S_{t}^{\alpha} \epsilon_{t-1}^{\alpha} \vee\left[\bigvee_{j \geq 2} a c \beta_{j-1}^{\alpha} S_{t}^{\alpha}\left(\prod_{i=0}^{j-2} S_{t-1-i}^{\alpha^{i+2}}\right) \epsilon_{t-j}^{\alpha^{j}}\right] \\
=c \epsilon_{t} \vee a c \beta_{0}^{\alpha} S_{t}^{\alpha} \epsilon_{t-1}^{\alpha} \vee\left[\bigvee_{j \geq 2} a c \beta_{j-1}^{\alpha}\left(\prod_{i=0}^{j-1} S_{t-i}^{\alpha^{i+1}}\right) \epsilon_{t-j}^{\alpha^{j}}\right] .
\end{aligned}
$$

Comparing all coefficients in the first formula and the last formula from the four formulas above, we have

$$
\beta_{0}=c, \beta_{j}=a c \beta_{j-1}^{\alpha}, j \geq 1 .
$$

It is possible to show (see the Appendix) that the distribution of $Z_{t, \alpha}$ defined by (8) and (9) coincides with the limiting marginal distribution of the $\mathrm{MAP}_{\alpha}(1)$ process (2) in Proposition 1. We summarize these arguments in Proposition 2.

Proposition 2. A $M A P_{\alpha}(1)$ process is causal, i.e. (8) holds.

\subsection{Temporal dependence among maxima}

To better understand the temporal structure of (2), we compute the following bivariate probability in Result \#1.

Result \#1.

$$
\begin{aligned}
& P\left(Z_{t, \alpha} \leq z_{t}, S_{t, \alpha} Z_{t-1, \alpha} \leq z_{t-1}\right) \\
& \quad=\exp \left(-\max \left\{\frac{d}{z_{t}}, \frac{c}{z_{t}}+\frac{d^{\alpha}}{z_{t-1}^{\alpha}}\right\}\right) .
\end{aligned}
$$

When $c=\alpha=1$ and $d=1 /(1-a)$, the formula above can be compared to model (1) from which we have

$$
\begin{aligned}
& P\left(Y_{t} \leq z_{t}, Y_{t-1} \leq z_{t-1}\right) \\
& \quad=\exp \left(-\max \left\{\frac{1}{(1-a) z_{t}}, \frac{1}{z_{t}}+\frac{1}{(1-a) z_{t-1}}\right\}\right) .
\end{aligned}
$$

It can be deduced as

$$
\begin{aligned}
& \lim _{\alpha \uparrow 1, c \uparrow 1} P\left(Z_{t, \alpha} \leq z_{t}, S_{t, \alpha} Z_{t-1, \alpha} \leq z_{t-1}\right) \\
& \quad=P\left(Y_{t} \leq z_{t}, Y_{t-1} \leq z_{t-1}\right) .
\end{aligned}
$$

This equation tells that the joint probability of $\left(Y_{t}, Y_{t-1}\right)$ can arbitrarily closely be approximated by the joint probability of the limit process of $\left(Z_{t, \alpha}, Z_{t-1, \alpha}\right)$.

The bivariate vector $\left(Y_{t}, Y_{t-1}\right)$ possesses the maxstability property

$$
\begin{aligned}
& P^{u}\left(Y_{t} \leq u z_{t}, Y_{t-1} \leq u z_{t-1}\right) \\
& \quad=P\left(Y_{t} \leq z_{t}, Y_{t-1} \leq z_{t-1}\right), \text { for any } u>0
\end{aligned}
$$

that represents the foundation of the Extreme Value Theory. Does this fundamental property still hold for the bivariate vector $\left(Z_{t, \alpha}, Z_{t-1, \alpha}\right)$ ? We can write that

$$
\begin{aligned}
& P^{u}\left(Z_{t, \alpha} \leq u z_{t}, S_{t, \alpha} Z_{t-1, \alpha} \leq u z_{t-1}\right) \\
& =\exp \left(-\max \left\{\frac{d}{z_{t}}, \frac{c}{z_{t}}+\frac{d^{\alpha} u^{1-\alpha}}{z_{t-1}^{\alpha}}\right\}\right)
\end{aligned}
$$

which implies that

$$
\begin{aligned}
\log \frac{P^{u}\left(Z_{t, \alpha} \leq u z_{t}, S_{t, \alpha} Z_{t-1, \alpha} \leq u z_{t-1}\right)}{P\left(Z_{t, \alpha} \leq z_{t}, S_{t, \alpha} Z_{t-1, \alpha} \leq z_{t-1}\right)} \\
\quad=\max \left[\frac{a c d^{\alpha}}{z_{t}}, \frac{d^{\alpha}}{z_{t-1}^{\alpha}}\right]-\max \left[\frac{a c d^{\alpha}}{z_{t}}, \frac{d^{\alpha} u^{1-\alpha}}{z_{t-1}^{\alpha}}\right] .
\end{aligned}
$$

An extension of max autoregressive models 255 

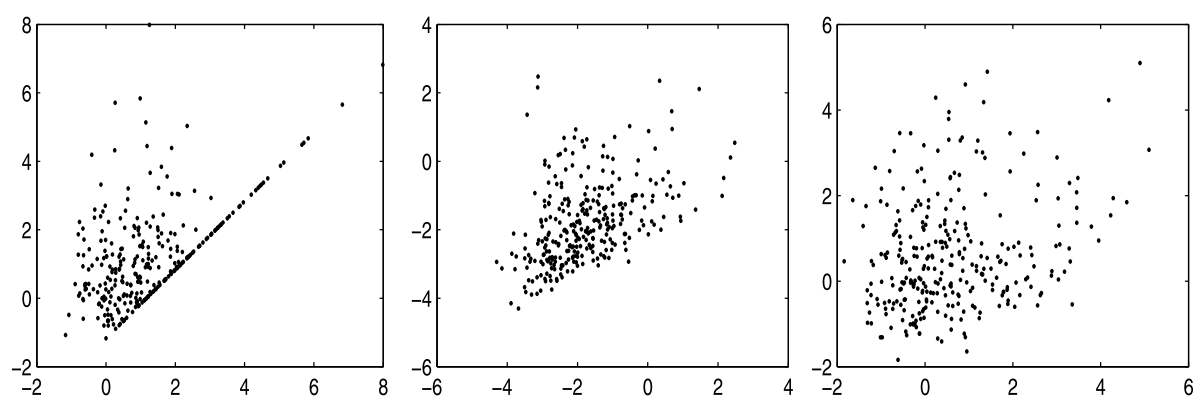

Figure 2. Illustrations of asymptotic dependence of $\log \left(Y_{t-1}\right)$ (X-axis) and $\log \left(Y_{t}\right)$ (Y-axis) in model (1) in the left panel, and asymptotic independence of $X_{t-1}\left(X\right.$-axis) and $X_{t}$ ( $Y$-axis) in model (4) with parameter values $a=0.3112, c=0.7325$, $\alpha=0.5020$. The middle panel is without max-shock noise, i.e. $\xi_{t}=-\infty$, while the right panel is with max-shock noise.

As $\alpha \uparrow 1, c \uparrow 1$, one can see that the bivariate vector $\left(Z_{t, \alpha}, Z_{t-1, \alpha}\right)$ becomes more and more max-stable.

To study the joint tail behavior, we examine the so called asymptotic dependence index introduced by [10]. We give the following definition.

Definition 1. Two identically distributed random variables $X$ and $Y$ with distribution function $F$ are called "asymptotically independent", if

$$
\lambda=\lim _{u \rightarrow x_{F}} P(Y>u \mid X>u)
$$

is 0 , where $x_{F}=\sup \{x \in \mathbb{R}: F(x)<1\}$. The quantity $\lambda$, if it exists, is called the bivariate asymptotic dependence index which quantifies the amount of dependence of the bivariate upper tails. If $\lambda>0$, then $X$ and $Y$ are called "asymptotically dependent."

The model (1) gives $\lambda=a$, i.e. $Y_{t}$ and $Y_{t-1}$ are asymptotically dependent. In viewing (4), one can see that $X_{t}$ and $X_{t-1}$ are more likely to be asymptotically independent mainly because the additive log-positive stable noise in $X_{t}$ is heavy tailed and is independent of $X_{t-1}$, and the max-shock noise is also independent of $X_{t-1}$. To prove the asymptotic (in)dependence of these two random variables, we may need to know the density of $S_{t}$. In Section 3, we will prove the asymptotic independence when $S_{t}$ is a positive $\alpha$-stable Lévy random variable. In Figure 2, we illustrate three simulated examples which are showing asymptotic (in)dependencies. The left panel depicts $\log \left(Y_{t-1}\right)$ and $\log \left(Y_{t}\right)$ in model (1) with parameter value $a=0.3112$. The middle panel is for model (4) with $a=0.3112, c=0.7325, \alpha=0.5020$, and without max-shock noise, i.e. $\xi_{t}=-\infty$. The right panel is for model (4) with $a=0.3112, c=0.7325, \alpha=0.5020$, and with max-shock noises.

From the left panel in Figure 2, one can clearly see the existence of a signature pattern, while the middle panel and the right panel do not show such kind of patterns. One can also see that model (2) can model a wide range of dependence in asymptotic independence.

\section{A SPECIAL CASE, THE MAP $1 / 2(1)$ PROCESS}

In the literature, Lévy distribution has been widely applied to many real data applications. A Lévy distributed random variable $S$ has the following density function:

$$
f_{L}(s, \delta)=\sqrt{\frac{\delta}{2 \pi}} e^{-\frac{\delta}{2 s}} \frac{1}{s^{3 / 2}}, \text { for all } s>0
$$

where $\delta$ is a positive parameter, and we denote $S \sim$ Lévy $(0, \delta)$. The following proposition shows that Lévy $(0, \delta)$ is positive $\alpha$-stable if and only if $\delta=\frac{1}{2}$.

Proposition 3. Suppose $S \sim \operatorname{Lévy}(0, \delta)$. Then $S$ is positive $\alpha$-stable if and only if $\delta=\alpha=\frac{1}{2}$.

For notational convenience, we drop the subindex $\alpha=$ $1 / 2$ in $Z_{t, 1 / 2}$, i.e. use $Z_{t}$, and the same is true for $S_{t}$. We also suppose that $Z_{t}$ is Fréchet distributed with scale parameter $d$, where $d$ satisfies the equation $a c d^{\frac{1}{2}}+c=d$.

\subsection{The tail dependence coefficient}

To study the dependence among extremes, $[8,9]$ introduced a very useful concept, the coefficient of tail dependence that provides a finer picture than the parameter $\lambda$. We now give a brief description of this coefficient denoted by $\eta$ characterized in Definition 1. For a broad range of joint distributions with unit Fréchet marginal variables, $[8,9]$ consider the following model

$$
P(X>u, Y>u) \sim \mathrm{L}\left(\frac{1}{P(X>u)}\right) P(X>u)^{1 / \eta}
$$

as $u \rightarrow \infty$, where $\mathrm{L}$ is a slowly varying function, i.e. $\mathrm{L}(t u) / \mathrm{L}(u) \rightarrow 1$ as $u \rightarrow \infty$ for any fixed $t>0$, and $\eta \in(0,1]$ is a constant. Using their terminology, the $\eta$ value effectively determines the decay rate of the joint bivariate survival function evaluated at the same large $u$, and $\eta$ is termed as the coefficient of tail dependence. Two marginal variables are called positively associated when $1 / 2<\eta \leq 1$; nearly independent when $\eta=1 / 2$; and negatively associated when 
$0<\eta<1 / 2$. Equation (11) can be expressed as

$$
P(Y>u \mid X>u) \sim \mathrm{L}\left(\frac{1}{P(X>u)}\right) P(X>u)^{1 / \eta-1}
$$

as $u \rightarrow \infty$, which shows how $\lambda$ changes with $\eta$. It is easy to see that the two variables $X$ and $Y$ are asymptotically dependent when $\eta=1$ and $\mathrm{L}(u) \nrightarrow 0$ as $u \rightarrow \infty$, and are asymptotically independent otherwise.

In our case, we would like to find $\eta$ of lag- 1 tail dependence for stationary $\mathrm{MAP}_{1 / 2}(1)$ process. Let $u$ be a threshold, we have $P\left(Z_{t} \leq u\right)=P\left(Z_{t-1} \leq u\right)=\exp (-d / u)$ and the following joint probabilities in Result \#2.

\section{Result \#2.}

$$
\begin{aligned}
P\left(Z_{t}\right. & \left.\leq u, Z_{t-1} \leq u\right) \\
= & \exp \left(-\frac{c}{u}\right)\left\{2 \exp \left(-\frac{d}{u}\right)-2 \exp \left(-\frac{d}{u}\right) \Phi\left(\frac{a c}{\sqrt{2 u}}\right)\right. \\
& -\exp \left(\frac{a c \sqrt{d}}{u}\right)\left[1-\Phi\left(\frac{a c+2 \sqrt{d}}{\sqrt{2 u}}\right)\right] \\
& \left.+\exp \left(-\frac{a c \sqrt{d}}{u}\right) \Phi\left(\frac{a c-2 \sqrt{d}}{\sqrt{2 u}}\right)\right\} .
\end{aligned}
$$

Then, we are able to calculate the following joint survival function of $\left(Z_{t-1}, Z_{t}\right)$ in the form of equation (11) in Result \#3.

\section{Result \#3.}

$$
\begin{aligned}
P\left(Z_{t}>u, Z_{t-1}>u\right) & \sim \frac{2 a c d}{\sqrt{\pi}} u^{-\frac{3}{2}} \\
& \sim \operatorname{cst} P\left(Z_{t}>u\right)^{1 / \eta} .
\end{aligned}
$$

Since $Z_{t}$ follows Fréchet distribution with scale parameter $d$, it is immediately from (14) that the coefficient of lag-1 tail dependence for stationary $\operatorname{MAP}_{1 / 2}(1)$ process is $\eta=2 / 3$ and the corresponding slowly varying function $L(u)=\frac{2 a c}{\sqrt{d \pi}}$. With $\eta=2 / 3$, we conclude that there exists positive dependence in $\mathrm{MAP}_{1 / 2}(1)$ processes and an asymptotic independence in the sense that $\lim P\left(Z_{t}>u \mid Z_{t-1}>u\right)=0$ as $u$ increases.

\subsection{Statistical estimation}

Although $\mathrm{MAP}_{\alpha}(1)$ processes have attractive probabilistic properties, there are difficulties in applying standard statistical estimation methods such as the maximum likelihood. This difficulty has been noted previously for M4 processes, the max-autoregressive moving average or MARMA processes of [3]. [6] got around this difficulty by defining a class of estimators based on empirical processes. [12] applied the generalized method of moment estimation to a class of sparse moving maxima models. The method proposed here for $\mathrm{MAP}_{\alpha}(1)$ models is similarly motivated.
In this section, we assume that the $\mathrm{MAP}_{1 / 2}(1)$ process is stationary. We first have the following results.

\section{Result \#4.}

$$
E\left(\exp \left\{-\frac{1}{Z_{t, \frac{1}{2}}}\right\}\right)=\frac{d}{d+1}
$$

and

$E\left(\exp \left\{-\frac{1}{Z_{t, \frac{1}{2}}}-\frac{1}{Z_{t-1, \frac{1}{2}}}\right\}\right)=\frac{c d+a c d \sqrt{d+1}}{(d+1)(c+1+a c \sqrt{d+1})}$

Using the stationary condition $a c \sqrt{d}+c=d$, we can construct generalized method of moments estimators for all three parameters. Our proposed estimator for $\theta=(d, a, c)$ is

$$
\begin{aligned}
\hat{\theta}= & \underset{\theta \in \mathbb{R}^{+} \times(0,1]^{2}}{\arg \min }\left[\left(\frac{1}{n} \sum_{i=1}^{n} \exp \left\{-\frac{1}{Z_{t+i, \frac{1}{2}}}\right\}-\frac{d}{d+1}\right)^{2}\right. \\
& +\left(\frac{1}{n} \sum_{i=1}^{n} \exp \left\{-\frac{1}{Z_{t+i, \frac{1}{2}}}-\frac{1}{Z_{t-1+i, \frac{1}{2}}}\right\}\right. \\
& \left.\left.-\frac{c d+a c d \sqrt{d+1}}{(d+1)(c+1+a c \sqrt{d+1})}\right)^{2}\right] .
\end{aligned}
$$

By ergodic theorem,

$$
\begin{gathered}
\frac{1}{n} \sum_{i=1}^{n} \exp \left\{-\frac{1}{Z_{t+i, \frac{1}{2}}}\right\} \stackrel{\text { a.s. }}{\rightarrow} \frac{d}{d+1}, \\
\frac{1}{n} \sum_{i=1}^{n} \exp \left\{-\frac{1}{Z_{t+i, \frac{1}{2}}}-\frac{1}{Z_{t-1+i, \frac{1}{2}}}\right\} \\
\stackrel{\text { a.s. }}{\rightarrow} \frac{c d+a c d \sqrt{d+1}}{(d+1)(c+1+a c \sqrt{d+1})} .
\end{gathered}
$$

Then by continuous mapping theorem, it is not difficult to see that our proposed estimators are strongly consistent. Due to the complexity of the joint distributions of $\left\{Z_{t, 1 / 2}\right\}$, the asymptotics of the estimators do not have an explicit form. In this work, we propose to report Monte Carlo confidence intervals for simulation examples and real data example as well.

\section{SIMULATION EXAMPLES}

In this section, we simulate the $\mathrm{MAP}_{1 / 2}(1)$ processes with different choices of parameters $a$ and $c$. It has been shown that for each set of parameter values, a generated process is a non-stationary process with its limit being stationary. Moreover, the process will reach its stationary limit quickly regardless of the initial value. Motivated from this fact, in each simulation, we discard the first 3,000 values of $Z_{t, \frac{1}{2}}$, and then simply treat the remaining simulated sequence as a stationary $\mathrm{MAP}_{1 / 2}(1)$ process. 
Table 1. Fitted parameter values and Monte Carlo confidence intervals for simulated data

\begin{tabular}{|c|c|c|c|c|c|c|c|}
\hline \hline & \multicolumn{3}{|c|}{$a=.8$} & \multicolumn{3}{c|}{$c=.8$} & $d=1.6128$ \\
\hline size & $95 \%$ M.C.I. & mean & median & $95 \%$ M.C.I. & mean & median & mean \\
\hline 1000 & $(.4964,1.0000)$ & .7890 & .7932 & $(.6838,1.0000)$ & .8148 & .8035 & 1.6149 \\
2000 & $(.5513,1.0000)$ & .7965 & .7980 & $(.6980, .9550)$ & .8077 & .8029 & 1.6139 \\
5000 & $(.6332,1.0000)$ & .8046 & .8005 & $(.7123, .8964)$ & .8002 & .7992 & 1.6123 \\
10000 & $(.6783, .9441)$ & .8029 & .8002 & $(.7329, .8691)$ & .7999 & .7996 & 1.6127 \\
\hline \multicolumn{6}{|c|}{$a=.2$} & \multicolumn{3}{c|}{$c=.2$} & $d=.2187$ \\
\hline size & $95 \%$ M.C.I. & mean & median & $95 \%$ M.C.I. & mean & median & mean \\
\hline 1000 & $(.0000, .5799)$ & .2124 & .1932 & $(.1660, .2324)$ & .1998 & .1998 & .2187 \\
2000 & $(.0033, .4570)$ & .2078 & .1998 & $(.1762, .2235)$ & .1998 & .1997 & .2187 \\
5000 & $(.0693, .3536)$ & .2017 & .1999 & $(.1851, .2154)$ & .2000 & .1999 & .2187 \\
10000 & $(.1043, .3089)$ & .2008 & .1999 & $(.1894, .2107)$ & .2000 & .2000 & .2187 \\
\hline \multicolumn{7}{|c|}{$a=.1$} & \multicolumn{2}{|c|}{$c=.9$} & & $d=.9895$ \\
\hline size & $95 \%$ M.C.I. & mean & median & $95 \%$ M.C.I. & mean & median & mean \\
\hline 1000 & $(.0000, .2418)$ & .1036 & .0967 & $(.7840,1.0000)$ & .8996 & .9009 & .9894 \\
2000 & $(.0219, .1938)$ & .1014 & .0991 & $(.8170, .9876)$ & .9005 & .9002 & .9897 \\
5000 & $(.0473, .1588)$ & .1000 & .0988 & $(.8465, .9558)$ & .9010 & .9010 & .9895 \\
10000 & $(.0619, .1415)$ & .1004 & .0998 & $(.8623, .9384)$ & .9000 & .8996 & .9896 \\
\hline
\end{tabular}

Table 2. Fitted parameter values and Monte Carlo confidence intervals for simulated data

\begin{tabular}{|c|c|c|c|c|c|c|c|}
\hline \hline & \multicolumn{3}{|c|}{$a=.9$} & \multicolumn{3}{c|}{$c=.1$} & $d=.1328$ \\
\hline size & $95 \%$ M.C.I. & mean & median & $95 \%$ M.C.I. & mean & median & mean \\
\hline 1000 & $(.2628,1.0000)$ & .7896 & .8999 & $(.0878, .1247)$ & .1038 & .1028 & .1332 \\
2000 & $(.4061,1.0000)$ & .8020 & .8999 & $(.0905, .1176)$ & .1023 & .1015 & .1328 \\
5000 & $(.5830,1.0000)$ & .8676 & .9000 & $(.0933, .1113)$ & .1010 & .1006 & .1328 \\
10000 & $(.6702,1.0000)$ & .8855 & .9000 & $(.0949, .1077)$ & .1005 & .1001 & .1328 \\
\hline \multicolumn{7}{|c|}{$a=.4$} & \multicolumn{3}{c|}{$c=.6$} & $d=.8169$ \\
\hline size & $95 \%$ M.C.I. & mean & median & $95 \%$ M.C.I. & mean & median & mean \\
\hline 1000 & $(.1996, .6867)$ & .4043 & .3934 & $(.4996, .7053)$ & .6021 & .6016 & .8170 \\
2000 & $(.2533, .5803)$ & .4036 & .3983 & $(.5295, .6747)$ & .6007 & .6000 & .8176 \\
5000 & $(.3045, .5089)$ & .3998 & .3976 & $(.5562, .6454)$ & .6007 & .6007 & .8169 \\
10000 & $(.3284, .4771)$ & .4001 & .3995 & $(.5691, .6334)$ & .6004 & .6005 & .8171 \\
\hline \multicolumn{7}{|c|}{$a=.6$} & \multicolumn{2}{c|}{$c=.4$} & & $d=.5833$ \\
\hline size & $95 \%$ M.C.I. & mean & median & $95 \%$ M.C.I. & mean & median & mean \\
\hline 1000 & $(.3097,1.0000)$ & .6088 & .5970 & $(.3320, .4788)$ & .4012 & .4006 & .5837 \\
2000 & $(.3906, .8648)$ & .6051 & .5958 & $(.3481, .4540)$ & .4010 & .4009 & .5843 \\
5000 & $(.4625, .7609)$ & .6015 & .5982 & $(.3661, .4343)$ & .4003 & .4003 & .5833 \\
10000 & $(.5004, .7155)$ & .6021 & .6000 & $(.3762, .4233)$ & .3998 & .3998 & .5832 \\
\hline
\end{tabular}

For each set of parameters $a, c$ and each pre-specified sample size, we simulate the process 5,000 times. By using the estimation method discussed in the previous section, we get the estimators $\hat{a}, \hat{c}$ for each simulated sequence. Tables 1 and 2 show the simulation results. In the tables, the size column shows the sample size after trimming the first 3,000 values, the M.C.I. column gives the lower limit and the upper limit of a 95\% Monte Carlo confidence interval based on 5,000 estimated values, the mean column shows the mean value of 5,000 estimated values, and similarly the median column shows the median of 5,000 estimated values.

From these two tables, one can see that the mean values and the median values are very close to their corresponding true parameter values, and the $95 \%$ Monte Carlo confidence intervals are short in length. These empirical evidences sug- gest that our proposed parameter estimators are consistent, and they well approximate true parameter values.

\section{REAL DATA APPLICATION}

In this section, we use river flow rate data to demonstrate the applications of our proposed new time series models. Particularly, we fit a $\mathrm{MAP}_{1 / 2}(1)$ model to deseasonalized weekly maxima of river flow rates of the Eagle River which is a tributary of the Colorado River, approximately 70 miles long, in west central Colorado. Our data source is the US Geological Survey (USGS). The site number of the Eagle is 09070000. "The Eagle rises in southeastern Eagle County, at the continental divide, and flows northwest past Gilman, Minturn and Avon. Near Wolcott, it turns 

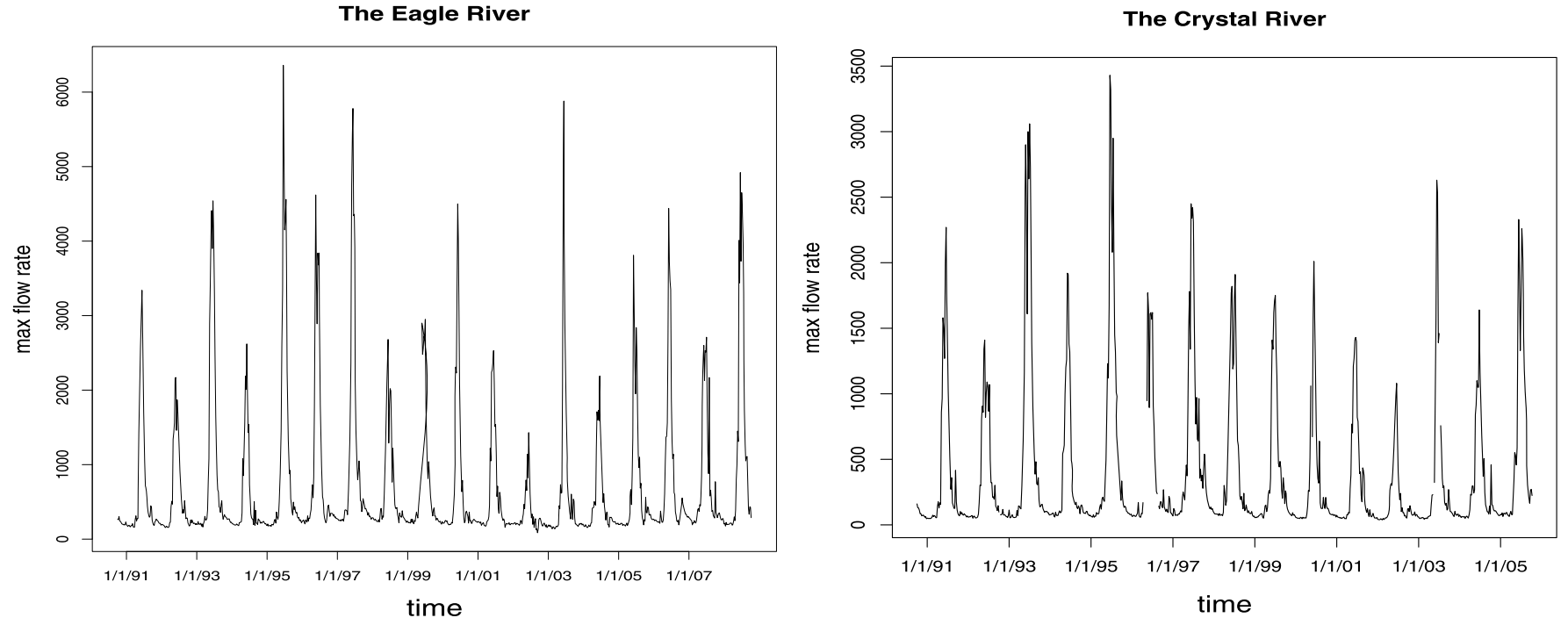

Figure 3. Weekly maxima of river flow rates $\left(\mathrm{ft}^{3} / \mathrm{sec}\right)$ in the Eagle River (left panel) and in the Crystal River (right panel).

Table 3. Fitted parameter values in $\operatorname{MAP}_{1 / 2}(1)$ with real data and Monte Carlo confidence intervals

\begin{tabular}{|c|c|c|c|c|}
\hline \hline & \multicolumn{2}{|c|}{ The Eagle River } & \multicolumn{2}{c|}{ The Crystal River } \\
\hline Parameter & Estimate & M.C.I & Estimate & M.C.I \\
\hline $\mathrm{a}$ & 1.0000 & $(.5594,1)$ & 1.0000 & $(.5809,1)$ \\
\hline $\mathrm{c}$ & .5903 & $(.5644, .7864)$ & .5987 & $(.5569, .7699)$ \\
\hline $\mathrm{d}$ & 1.2504 & & 1.2745 & \\
\hline
\end{tabular}

west, flowing past Eagle and Gypsum, and joins the Colorado at Dotsero, in western Eagle County. The Eagle is navigable by typical small river craft upstream to Vail in most of the time. Its flow ranges from $200 \mathrm{cfs}$ in late summer of dry years to $7,000 \mathrm{cfs}$ during spring runoff (Wikipedia http://en.wikipedia.org/wiki/Eagle_River_(Colorado))."

The Eagle River flow rates show very strong seasonal effects (Figure 3), most notably, snowmelt in the early summer. This phenomenon is typical within rivers in Colorado geographic area, for example the Crystal River with which [1] analyzed the maximum weekly flow rates using approximated conditional density approach for the deseasonalized data. For comparison purpose, we also include the Crystal River data (the same as used in [1] in this analysis), and we analyze the deseasonalized weekly maxima of flow rates and adopt the same procedure of deseasonalization as done in [1]. Next, we describe the deseasonalization procedure for the Eagle River flow rates.

We first obtain from the USGS Instantaneous Data Archive (IDA) a time series data set (ranging from 199010-01 to 2008-09-30 with one reading every 15 minutes). There are about 166 days in which the IDA has not recorded flow rates. Second, from the USGS National Water Information System (NWIS), we obtain daily mean flow rates (ranging from 1946-10-01 to 2008-09-30) with no missing records. Third, for each data point in the IDA time series, we transform the data using the following three steps: 1) compute the mean value from the NWIS data across all years within a thirteen-day local window which is centered on the day the IDA data point value is recorded; 2) compute the standard deviation from the NWIS data within all the same local windows; 3 ) standardize the IDA data point value using the mean and the standard deviation obtained in 1) and 2). Fourth, we obtain a deseasonalized daily IDA time series after taking the maximum of all standardized daily point values for each day. Fifth, we use a simple regression model to "recover" the missing daily maxima by regressing the maxima on the means over the period of 1990-10-01 to 2008-09-30. The squared regression coefficient $R^{2}=.998$ which shows a very good fit. Finally, we obtain the weekly maxima and plot them in Figures 4 (left panel) and 5 (left panel).

We note that Figure 4 is comparable to Figure 1 . The same comparison holds for the Crystal River deseasonalized weekly maxima of river flow rates directly. Fitting model (4) to the deseasonalized weekly maxima, we obtain the following fitted values in Table 3 . We note that the estimated values of the parameter $a$ are 1 in both cases. This can be understood by thinking that the parameter $a$ is combined into $c$, and we still have $a c<1$, i.e. two processes are stationary.

Using the estimated values in the table, we simulate weekly maximum deseasonalized time series and compare 

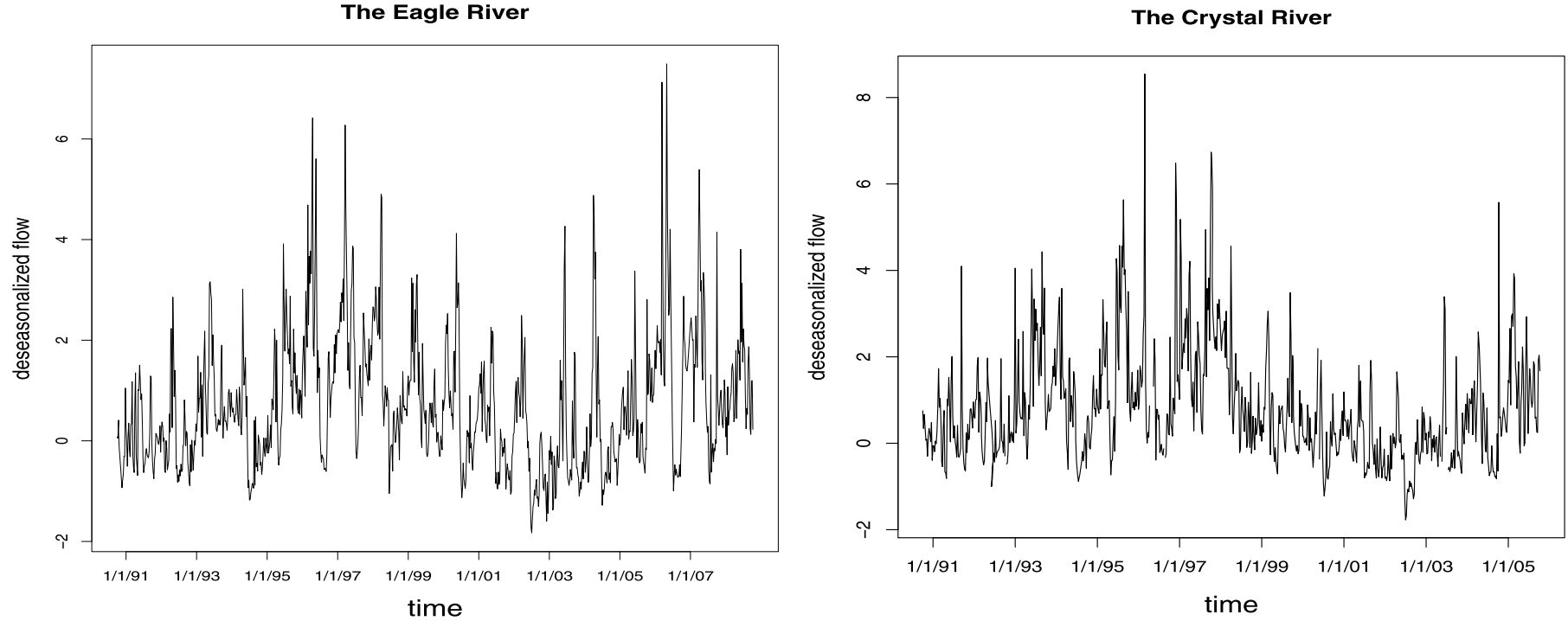

Figure 4. Deseasonalized weekly maxima of river flow rates $\left(\mathrm{ft}^{3} / \mathrm{sec}\right)$ in the Eagle River (left panel) and in the Crystal River (right panel).
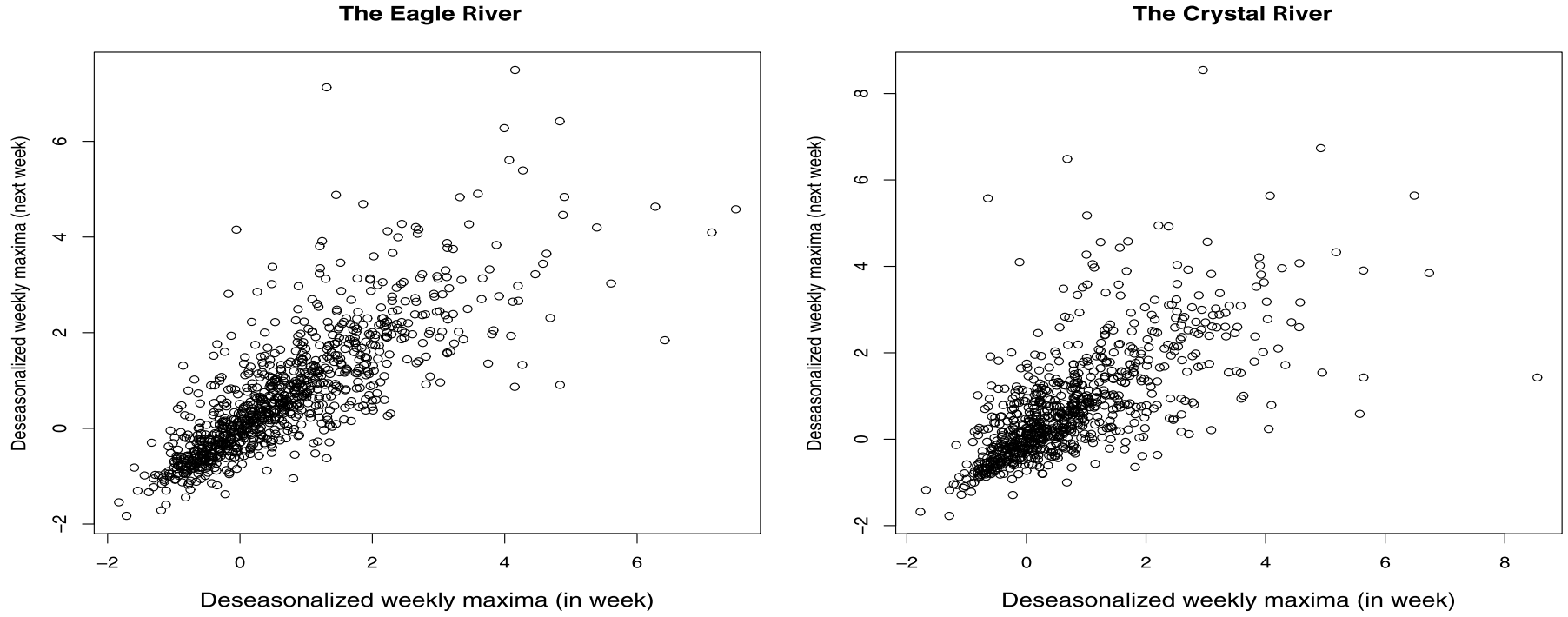

Figure 5. Deseasonalized weekly maxima of river flow rates $\left(\mathrm{ft}^{3} / \mathrm{sec}\right)$ in the Eagle River (left panel) and in the Crystal River (right panel).

the simulated values with the observed values using quantilequantile plots. In Figure 6, we can see that our proposed models reasonably well approximate the weekly maxima, especially in the tails.

\section{CONCLUSIONS}

Modeling extremes in time series is challenging. In this paper, we have generalized the max-autoregressive processes considered in [3] to the max autoregressive processes with positive alpha stable coefficients and unit Fréchet shocks, i.e. $\mathrm{MAP}_{\alpha}(p)$ models which eliminate the abnormally signature patterns. We have proved that $\mathrm{MAP}_{1 / 2}(1)$ models asymptotically independent time series, and empirically demonstrated that $\mathrm{MAP}_{\alpha}(p)$ models asymptotically independent time series, which enriches models for extremes in time series. Our data examples suggest that $\mathrm{MAP}_{1 / 2}(1)$ models can directly be applied to some deseasonalized river flow rate data, which is certainly useful in hydrological study and river engineering management.

There are several future emerging research directions related to our present work. As mentioned in the introduction section, there are variants of our proposed models. The major challenges in our proposed models and other potential 

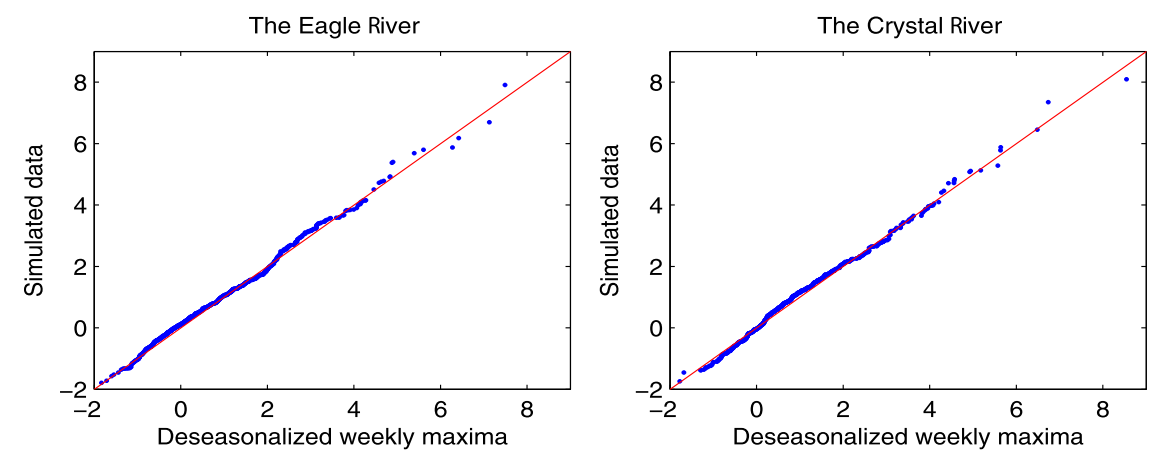

Figure 6. Quantile-quantile plots for deseasonalized weekly maxima of river flow rates ( $\mathrm{ft}^{3} / \mathrm{sec}$ ) in the Eagle River (left panel) and in the Crystal River (right panel).

extensions are to study model reducibility, causality and predictability as they have been extensively studied in ARMA models. Constructing efficient parameter estimation methods is still a challenging task, e.g. when $\alpha$ is also unknown. We expect that there will be more and more theoretical developments in this field. We also expect more and more applications of these new models to different research fields in the near future.

\section{APPENDIX}

Proof of Proposition 1. Note that $\log Z_{0, \alpha}$ is Gumbel with $\log \left(b_{0}\right)$ as the location parameter and one as the scale parameter. Then, $\alpha \log S_{1, \alpha}+\alpha \log Z_{0, \alpha}$ follows a Gumbel distribution with $\alpha \log \left(b_{0}\right)$ as the location parameter and one as the scale parameter. We have

$$
\begin{aligned}
P & \left(Z_{1, \alpha} \leq z\right)=P\left(S_{1, \alpha} Z_{0, \alpha} \leq(z / a c)^{1 / \alpha}\right) P\left(\epsilon_{1} \leq z / c\right), \\
& =P\left\{\alpha \log S_{1, \alpha}+\alpha \log Z_{0, \alpha} \leq \log \left(\frac{z}{a c}\right)\right\} \times \exp \left(-\frac{c}{z}\right), \\
& =\exp \left\{-\exp \left[-\log \left(\frac{z}{a c}\right)-\alpha \log \left(b_{0}\right)\right]\right\} \times \exp \left(-\frac{c}{z}\right) \\
& =\exp \left(-\frac{a c b_{0}^{\alpha}+c}{z}\right),
\end{aligned}
$$

and similarly we have

$$
P\left(Z_{t, \alpha} \leq z\right)=\exp \left(-\frac{a c b_{t-1}^{\alpha}+c}{z}\right),
$$

for $t=1,2, \ldots$ The proof of $\left\{b_{t}\right\}$ having a limit $d$ is divided into three steps.

First, it is easy to see that the function $g(x)=x-a c x^{\alpha}-c$ is decreasing when $0<x<(a c \alpha)^{1 /(1-\alpha)}$, and is increasing when $x>(a c \alpha)^{1 /(1-\alpha)}$. In addition, $g(x)$ has a unique zero in $\mathbb{R}^{+}$, denoted by $d, g(x)>0$ when $x>d$, and $g(x)<0$ when $0<x<d$.

Second, let's assume that for the initial condition $b_{0}$ we have $b_{0} \geq d$. Then by $g\left(b_{0}\right) \geq 0$,

$$
\begin{aligned}
& b_{0} \geq b_{1}=a c b_{0}^{\alpha}+c \geq a c d^{\alpha}+c=d, \\
& b_{1} \geq b_{2}=a c b_{1}^{\alpha}+c \geq a c d^{\alpha}+c=d .
\end{aligned}
$$

Continuing this procedure, we have that $\left\{b_{t}\right\}$ is a decreasing sequence with lower bound $d$, and hence it has a limit. Similarly, let's assume that for the initial condition $b_{0}$ we have $b_{0}<d$. Then by $g\left(b_{0}\right)<0$,

$$
\begin{aligned}
& b_{0}<b_{1}=a c b_{0}^{\alpha}+c<a c d^{\alpha}+c=d, \\
& b_{1}<b_{2}=a c b_{1}^{\alpha}+c<a c d^{\alpha}+c=d .
\end{aligned}
$$

Continuing this procedure, we have that $\left\{b_{t}\right\}$ is an increasing sequence with upper bound $d$, and hence it has a limit.

Third, let $t$ tend to infinity on the both sides of $b_{t}=$ $a c b_{t-1}^{\alpha}+c$. Then,

$$
\begin{array}{r}
\lim _{t \rightarrow \infty} b_{t}=\lim _{t \rightarrow \infty}\left\{a c b_{t-1}^{\alpha}+c\right\} \\
\Rightarrow b=a c b^{\alpha}+c
\end{array}
$$

By the uniqueness of zero of $g(x)$, the limit $b$ must be equal to $d$, which satisfies $a c d^{\alpha}+c=d$.

For the second part of the proposition, we assume $b_{0}=d$. First, when $t=0, P\left(Z_{0, \alpha} \leq z_{0}\right)=P\left(Z_{k, \alpha} \leq z_{0}\right)$ for any $k \geq$ 0 , because the marginal distribution is identical to Fréchet distribution with scale parameter $d$.

Next, assume that for $t=n \geq 0$, we have for any constant values $z_{0}, z_{1}, \ldots$

$$
\begin{aligned}
& P\left(Z_{0, \alpha} \leq z_{0}, \ldots, Z_{n, \alpha} \leq z_{n}\right) \\
& \quad=P\left(Z_{k, \alpha} \leq z_{0}, \ldots, Z_{k+n, \alpha} \leq z_{n}\right)
\end{aligned}
$$

for any $k \geq 0$. Then for $t=n+1$,

$$
\begin{aligned}
& P\left(Z_{0, \alpha} \leq z_{0}, \ldots, Z_{n, \alpha} \leq z_{n}, Z_{n+1, \alpha} \leq z_{n+1}\right) \\
& =P\left(Z_{0, \alpha} \leq z_{0}, \ldots, Z_{n, \alpha} \leq z_{n}, Z_{n, \alpha} \leq \frac{z_{n+1}^{1 / \alpha}}{(a c)^{1 / \alpha} S_{n+1, \alpha}}\right. \\
& \left.\quad \epsilon_{n+1} \leq \frac{z_{n+1}}{a}\right)
\end{aligned}
$$




$$
\begin{aligned}
= & E\left[P \left(\left\{Z_{0, \alpha} \leq z_{0}, \ldots, Z_{n, \alpha} \leq \min \left(z_{n}, \frac{z_{n+1}^{1 / \alpha}}{(a c)^{1 / \alpha} S_{n+1, \alpha}}\right)\right\}\right.\right. \\
& \left.\left.\mid S_{n+1, \alpha}\right)\right] P\left(\epsilon_{n+1} \leq \frac{z_{n+1}}{a}\right) \\
= & E\left[P \left(\left\{Z_{k, \alpha} \leq z_{0}, \ldots,\right.\right.\right. \\
& \left.\left.\left.Z_{k+n, \alpha} \leq \min \left(z_{n}, \frac{z_{n+1}^{1 / \alpha}}{(a c)^{1 / \alpha} S_{k+n+1, \alpha}}\right)\right\} \mid S_{k+n+1, \alpha}\right)\right] \\
& P\left(\epsilon_{k+n+1} \leq \frac{z_{n+1}}{a}\right) \\
= & P\left(Z_{k, \alpha} \leq z_{0}, \ldots, Z_{k+n, \alpha} \leq z_{n},\right. \\
& \left.Z_{k+n, \alpha} \leq \frac{z_{n+1}^{1 / \alpha}}{(a c)^{1 / \alpha} S_{k+n+1, \alpha}}, \epsilon_{k+n+1} \leq \frac{z_{n+1}}{a}\right) \\
= & P\left(Z_{k, \alpha} \leq z_{0}, \ldots, Z_{k+n, \alpha} \leq z_{n}, Z_{k+n+1, \alpha} \leq z_{n+1}\right)
\end{aligned}
$$

$$
\begin{aligned}
& =\exp \left(-\frac{\beta_{0}}{z}\right) E\left[\lim _{n \rightarrow \infty} \prod_{j=1}^{n} \exp \left(-\frac{\beta_{j}^{\frac{1}{\alpha^{j}}} \prod_{i=0}^{j-1} S_{t-i}^{\frac{1}{\alpha^{j-i-1}}}}{z^{\frac{1}{\alpha^{j}}}}\right)\right] \\
& \quad \equiv \exp \left(-\frac{\beta_{0}}{z}\right) E\left[\lim _{n \rightarrow \infty} \prod_{j=1}^{n} F_{j}\right] .
\end{aligned}
$$

Since $0 \leq F_{j} \leq 1, \forall j \geq 1,\left\{\prod_{j=1}^{n} F_{j}\right\}$ is a non-increasing random variable sequence which is bounded by 1 . By dominated convergence theorem, we have

$$
\begin{aligned}
& (16)=\exp \left(-\frac{\beta_{0}}{z}\right) \lim _{n \rightarrow \infty} E\left[\prod_{j=1}^{n} F_{j}\right] \\
& =\lim _{n \rightarrow \infty} \exp \left(-\frac{\beta_{0}}{z}\right) \\
& E\left\{E \left[\left(\prod_{j=1}^{n-1} F_{j}\right) \exp \left(-\frac{\beta_{n}^{\frac{1}{\alpha^{n}}} \prod_{i=0}^{n-1} S_{t-i}^{\frac{1}{\alpha^{n-i-1}}}}{z^{\frac{1}{\alpha^{n}}}}\right)\right.\right. \\
& \left.\left.\mid S_{t}, S_{t-1}, \ldots, S_{t-n+2}\right]\right\} \\
& =\lim _{n \rightarrow \infty} \exp \left(-\frac{\beta_{0}}{z}\right) \\
& E\left\{( \prod _ { j = 1 } ^ { n - 1 } F _ { j } ) E \left[\exp \left(-\frac{\beta_{n}^{\frac{1}{\alpha^{n}}} \prod_{i=0}^{n-1} S_{t-i}^{\frac{1}{\alpha^{n-i-1}}}}{z^{\frac{1}{\alpha^{n}}}}\right)\right.\right. \\
& \left.\left.\mid S_{t}, S_{t-1}, \ldots, S_{t-n+2}\right]\right\} \\
& =\lim _{n \rightarrow \infty} \exp \left(-\frac{\beta_{0}}{z}\right) \\
& E\left\{\left(\prod_{j=1}^{n-2} F_{j}\right) \exp \left(-\frac{\beta_{n-1}^{\frac{1}{\alpha^{n-1}}} \prod_{i=0}^{n-2} S_{t-i}^{\frac{1}{\alpha^{n-i-2}}}}{z^{\frac{1}{\alpha^{n-1}}}}\right)\right. \\
& \left.\exp \left(-\frac{\beta_{n}^{\frac{1}{\alpha^{n-1}}} \prod_{i=0}^{n-2} S_{t-i}^{\frac{1}{\alpha^{n-i-2}}}}{z^{\frac{1}{\alpha^{n-1}}}}\right)\right\} \\
& =\lim _{n \rightarrow \infty} \exp \left(-\frac{\beta_{0}}{z}\right) E\left\{\left(\prod_{j=1}^{n-2} F_{j}\right)\right. \\
& \left.\exp \left(-\frac{\left(\beta_{n-1}^{\frac{1}{\alpha^{n-1}}}+\beta_{n}^{\frac{1}{\alpha^{n-1}}}\right) \prod_{i=0}^{n-2} S_{t-i}^{\frac{1}{\alpha^{n-i-2}}}}{z^{\frac{1}{\alpha^{n-1}}}}\right)\right\} \text {. }
\end{aligned}
$$

Using this conditional expectation argument repeatedly, we have

$$
\begin{aligned}
(17)= & \lim _{n \rightarrow \infty} \exp \left(-\frac{\beta_{0}}{z}\right) E\left\{( \prod _ { j = 1 } ^ { n - 3 } F _ { j } ) \operatorname { e x p } \left(-z^{-\frac{1}{\alpha^{n-2}}}\right.\right. \\
& {\left.\left.\left[\beta_{n-2}^{\frac{1}{\alpha^{n-2}}}+\left(\beta_{n-1}^{\frac{1}{\alpha^{n-1}}}+\beta_{n}^{\frac{1}{\alpha^{n-1}}}\right)^{\alpha}\right] \prod_{i=0}^{n-3} S_{t-i}^{\frac{1}{\alpha^{n-i-3}}}\right)\right\} }
\end{aligned}
$$$$
\left.\exp \left(-\frac{\beta_{2}^{\frac{1}{\alpha^{2}}} S_{t}^{\frac{1}{\alpha}} S_{t-1}}{z^{\frac{1}{\alpha^{2}}}}\right) \exp \left(-\frac{\beta_{3}^{\frac{1}{\alpha^{3}}} S_{t}^{\frac{1}{\alpha^{2}}} S_{t-1}^{\frac{1}{\alpha}} S_{t-2}}{z^{\frac{1}{\alpha^{3}}}}\right) \cdots\right]
$$

262 P. Naveau, Z. Zhang and B. Zhu 


$$
\begin{aligned}
= & \lim _{n \rightarrow \infty} \exp \left(-\frac{\beta_{0}}{z}\right) E\left[\operatorname { e x p } \left(-z^{-\frac{1}{\alpha}}\left\{\beta_{1}^{\frac{1}{\alpha}}+\left\{\beta_{2}^{\frac{1}{\alpha^{2}}}+\left\{\beta_{3}^{\frac{1}{\alpha^{3}}}\right.\right.\right.\right.\right. \\
& \left.\left.\left.\left.\left.+\cdots\left\{\beta_{n-2}^{\frac{1}{\alpha^{n-2}}}+\left\{\beta_{n-1}^{\frac{1}{\alpha^{n-1}}}+\beta_{n}^{\frac{1}{\alpha^{n-1}}}\right\}^{\alpha}\right\}^{\alpha} \cdots\right\}^{\alpha}\right\}^{\alpha}\right\} S_{t}\right)\right] \\
= & \lim _{n \rightarrow \infty} \exp \left(-\frac{1}{z}\left[\beta_{0}+\left\{\beta_{1}^{\frac{1}{\alpha}}+\left\{\beta_{2}^{\frac{1}{\alpha^{2}}}+\left\{\beta_{3}^{\frac{1}{\alpha^{3}}}\right.\right.\right.\right.\right. \\
& \left.\left.\left.\left.\left.+\cdots\left\{\beta_{n-2}^{\frac{1}{\alpha^{n-2}}}+\left\{\beta_{n-1}^{\frac{1}{\alpha^{n-1}}}+\beta_{n}^{\frac{1}{\alpha^{n-1}}}\right\}^{\alpha}\right\}^{\alpha} \cdots\right\}^{\alpha}\right\}^{\alpha}\right\}^{\alpha}\right]\right) \\
\equiv & \lim _{n \rightarrow \infty} \exp \left(-\frac{d_{n}}{z}\right)=\exp \left(-\frac{\lim _{n \rightarrow \infty} d_{n}}{z}\right) .
\end{aligned}
$$

Using the recursions (9) that $\left\{\beta_{j}\right\}$ satisfies, we can show that $\left\{d_{n}\right\}$ is a non-decreasing sequence with finite limit $d$. Hence, $Z_{t, \alpha}$ follows Fréchet distribution with scale parameter $d=$ $\beta_{0}+\left\{\beta_{1}^{\frac{1}{\alpha}}+\left\{\beta_{2}^{\frac{1}{\alpha^{2}}}+\left\{\beta_{3}^{\frac{1}{\alpha^{3}}}+\cdots\right\}^{\alpha}\right\}^{\alpha}\right\}^{\alpha}$, which satisfies

$$
\begin{aligned}
a c d^{\alpha} & +c \\
= & c+a c\left\{\beta_{0}+\left\{\beta_{1}^{\frac{1}{\alpha}}+\left\{\beta_{2}^{\frac{1}{\alpha^{2}}}\right.\right.\right. \\
& \left.\left.\left.+\left\{\beta_{3}^{\frac{1}{\alpha^{3}}}+\cdots\right\}^{\alpha}\right\}^{\alpha}\right\}^{\alpha}\right\}^{\alpha} \\
= & \beta_{0}+\left\{a^{\frac{1}{\alpha}} c^{\frac{1}{\alpha}} \beta_{0}+a^{\frac{1}{\alpha}} c^{\frac{1}{\alpha}}\left\{\beta_{1}^{\frac{1}{\alpha}}+\left\{\beta_{2}^{\frac{1}{\alpha^{2}}}\right.\right.\right. \\
& \left.\left.\left.+\left\{\beta_{3}^{\frac{1}{\alpha^{3}}}+\cdots\right\}^{\alpha}\right\}^{\alpha}\right\}^{\alpha}\right\}^{\alpha} \\
= & \beta_{0}+\left\{\beta_{1}^{\frac{1}{\alpha}}+\left\{a^{\frac{1}{\alpha^{2}}} c^{\frac{1}{\alpha^{2}}} \beta_{1}^{\frac{1}{\alpha}}+a^{\frac{1}{\alpha^{2}}} c^{\frac{1}{\alpha^{2}}}\left\{\beta_{2}^{\frac{1}{\alpha^{2}}}\right.\right.\right. \\
& \left.\left.\left.+\left\{\beta_{3}^{\frac{1}{\alpha^{3}}}+\cdots\right\}^{\alpha}\right\}^{\alpha}\right\}^{\alpha}\right\}^{\alpha} \\
= & \beta_{0}+\left\{\beta_{1}^{\frac{1}{\alpha}}+\left\{\beta_{2}^{\frac{1}{\alpha^{2}}}+\left\{a^{\frac{1}{\alpha^{3}}} c^{\frac{1}{\alpha^{3}}} \beta_{2}^{\frac{1}{\alpha^{2}}}\right.\right.\right. \\
& \left.\left.\left.+a^{\frac{1}{\alpha^{3}}} c^{\frac{1}{\alpha^{3}}}\left\{\beta_{3}^{\frac{1}{\alpha^{3}}}+\cdots\right\}^{\alpha}\right\}^{\alpha}\right\}^{\alpha}\right\}^{\alpha} \\
= & \cdots \\
= & \beta_{0}+\left\{\beta_{1}^{\frac{1}{\alpha}}+\left\{\beta_{2}^{\frac{1}{\alpha^{2}}}+\left\{\beta_{3}^{\frac{1}{\alpha^{3}}}+\cdots\right\}^{\alpha}\right\}^{\alpha}\right\}^{\alpha} \\
= & d .
\end{aligned}
$$

Actually, we have shown that the distribution of $Z_{t, \alpha}$ coincides with the limiting marginal distribution of the $\mathrm{MAP}_{\alpha}(1)$ process (2) in Proposition 1, which implies that the relation (8) is true.

Proof of Result \#1.

$$
\begin{aligned}
P\left(Z_{t, \alpha} \leq z_{t}, S_{t, \alpha} Z_{t-1, \alpha} \leq z_{t-1}\right) & = \\
= & P\left(\epsilon_{t} \leq z_{t} / c\right) P\left(S_{t, \alpha} Z_{t-1, \alpha} \leq m_{t}\right) \\
& \quad \text { with } m_{t}=\min \left(\left(z_{t} / a c\right)^{1 / \alpha}, z_{t-1}\right) \\
= & \exp \left(-c / z_{t}\right) \int P\left(Z_{t-1} \leq m_{t} / s\right) f_{S}(s) d s
\end{aligned}
$$

with $f_{S}$ the density of $S_{t}$,

$$
=\exp \left(-c / z_{t}\right) \int \exp \left(-s d / m_{t}\right) f_{S}(s) d s,
$$

because $Z_{t-1}$ is Fréchet,

$$
=\exp \left(-c / z_{t}\right) E\left[\exp \left(-S_{t} d / m_{t}\right)\right] \text {, }
$$

$$
\begin{aligned}
& =\exp \left(-c / z_{t}\right) \exp \left[-\left(d / m_{t}\right)^{\alpha}\right], \text { because of }(3), \\
& =\exp \left(-c / z_{t}\right) \exp \left[-d^{\alpha} \max \left\{a c / z_{t}, 1 / z_{t-1}^{\alpha}\right\}\right], \\
& =\exp \left(-\max \left\{\frac{a c d^{\alpha}+c}{z_{t}}, \frac{c}{z_{t}}+\frac{d^{\alpha}}{z_{t-1}^{\alpha}}\right\}\right), \\
& =\exp \left(-\max \left\{\frac{d}{z_{t}}, \frac{c}{z_{t}}+\frac{d^{\alpha}}{z_{t-1}^{\alpha}}\right\}\right) .
\end{aligned}
$$

Proof of Proposition 3. Taking the Laplace transform of $S$, we have

$$
E(\exp (-u S))=\sqrt{\frac{c}{2 \pi}} \int_{0}^{\infty} e^{-u s-\frac{c}{2 s}} \frac{1}{s^{3 / 2}} d s
$$

for all $u \geq 0$. In order to calculate the integral in the righthand side of (18), let $w=\sqrt{u s}+\sqrt{\frac{c}{2 s}}$ and $v=\sqrt{u s}-\sqrt{\frac{c}{2 s}}$. Then,

$$
\begin{aligned}
\int_{0}^{\infty} & e^{-u s-\frac{c}{2 s}} \frac{1}{s^{3 / 2}} d s \\
= & -\frac{2}{\sqrt{c / 2}} \int_{0}^{\infty} e^{-u s-\frac{c}{2 s}}\left(-\frac{\sqrt{c / 2}}{2 s^{3 / 2}}+\frac{\sqrt{u}}{2 \sqrt{s}}\right) d s \\
& +\frac{2}{\sqrt{c / 2}} \int_{0}^{\infty} e^{-u s-\frac{c}{2 s}} \frac{\sqrt{u}}{2 \sqrt{s}} d s \\
= & -\frac{2}{\sqrt{c / 2}} \int_{\infty}^{\infty} e^{-w^{2}+\sqrt{2 u c}} d w+\sqrt{\frac{2 u}{c}} \int_{0}^{\infty} e^{-u s-\frac{c}{2 s}} \frac{1}{\sqrt{s}} d s \\
= & \sqrt{\frac{2 u}{c}} \int_{0}^{\infty} e^{-u s-\frac{c}{2 s}} \frac{1}{\sqrt{s}} d s,
\end{aligned}
$$

and

$$
\begin{aligned}
\int_{0}^{\infty} & e^{-u s-\frac{c}{2 s}} \frac{1}{s^{3 / 2}} d s \\
= & \frac{2}{\sqrt{c / 2}} \int_{0}^{\infty} e^{-u s-\frac{c}{2 s}}\left(\frac{\sqrt{c / 2}}{2 s^{3 / 2}}+\frac{\sqrt{u}}{2 \sqrt{s}}\right) d s \\
& -\frac{2}{\sqrt{c / 2}} \int_{0}^{\infty} e^{-u s-\frac{c}{2 s}} \frac{\sqrt{u}}{2 \sqrt{s}} d s \\
= & \frac{2}{\sqrt{c / 2}} \int_{-\infty}^{\infty} e^{-v^{2}-\sqrt{2 u c}} d v-\sqrt{\frac{2 u}{c}} \int_{0}^{\infty} e^{-u s-\frac{c}{2 s}} \frac{1}{\sqrt{s}} d s
\end{aligned}
$$

which gives

$$
\begin{aligned}
\int_{0}^{\infty} e^{-u s-\frac{c}{2 s}} \frac{1}{s^{3 / 2}} d s & =\frac{1}{\sqrt{c / 2}} \int_{-\infty}^{\infty} e^{-v^{2}-\sqrt{2 u c}} d v \\
& =\frac{e^{-\sqrt{2 u c}}}{\sqrt{c / 2}} \int_{-\infty}^{\infty} e^{-v^{2}} d v \\
& =\sqrt{\frac{2 \pi}{c}} e^{-\sqrt{2 u c}}
\end{aligned}
$$

Plugging (19) into the right-hand side of (18), we have

An extension of max autoregressive models 263 


$$
\begin{aligned}
E(\exp (-u S)) & =\sqrt{\frac{c}{2 \pi}} \int_{0}^{\infty} e^{-u s-\frac{c}{2 s}} \frac{1}{s^{3 / 2}} d s \\
& =\sqrt{\frac{c}{2 \pi}} \sqrt{\frac{2 \pi}{c}} e^{-\sqrt{2 u c}}=e^{-\sqrt{2 u c}} .
\end{aligned}
$$

Hence, $S$ is positive $\alpha$-stable if and only if $c=\frac{1}{2}$, which implies $\alpha=\frac{1}{2}$.

Proof of Result \#2. Recall that $S_{t}$ is $\operatorname{Lévy}\left(0, \frac{1}{2}\right)$ distributed and it is possible to show that

$$
P\left(S_{t} \leq v\right)=2\left[1-\Phi\left(\frac{1}{\sqrt{2 v}}\right)\right],
$$

where $\Phi(\cdot)$ is the standard normal cdf of $\mathrm{N}(0,1)$. Then

$$
\begin{aligned}
P\left(Z_{t} \leq u, Z_{t-1} \leq u\right) & \\
= & P\left(\max \left\{a\left(S_{t} Z_{t-1}\right)^{0.5}, \epsilon_{t}\right\} \leq \frac{u}{c}, Z_{t-1} \leq u\right) \\
= & P\left(S_{t} Z_{t-1} \leq\left(\frac{u}{a c}\right)^{2}, \epsilon_{t} \leq \frac{u}{c}, Z_{t-1} \leq u\right) \\
= & P\left(\epsilon_{t} \leq \frac{u}{c}\right) P\left(S_{t} Z_{t-1} \leq\left(\frac{u}{a c}\right)^{2}, Z_{t-1} \leq u\right) \\
= & \exp \left(-\frac{c}{u}\right) E\left[P\left(S_{t} Z_{t-1} \leq\left(\frac{u}{a c}\right)^{2}, Z_{t-1} \leq u \mid Z_{t-1}\right)\right] \\
= & \exp \left(-\frac{c}{u}\right) \int_{0}^{u} P\left(S_{t} \leq \frac{(u / a c)^{2}}{y}\right) g_{Z_{t-1}}(y) d y \\
& \operatorname{with} g_{Z_{t-1}}(y)=\frac{d}{y^{2}} \exp \left(-\frac{d}{y}\right) \\
= & 2 \exp \left(-\frac{c}{u}\right) \int_{0}^{u}\left[1-\Phi\left(\frac{a c \sqrt{y}}{u \sqrt{2}}\right)\right] g_{Z_{t-1}}(y) d y
\end{aligned}
$$

by $(20)$,

$$
\begin{aligned}
& =2 \exp \left(-\frac{c}{u}\right) \exp \left(-\frac{d}{u}\right) \\
(21) & -2 \exp \left(-\frac{c}{u}\right) \int_{0}^{u} \Phi\left(\frac{a c \sqrt{y}}{u \sqrt{2}}\right) \frac{d}{y^{2}} \exp \left(-\frac{d}{y}\right) d y .
\end{aligned}
$$

The integral in equation (21) can be expressed as

$$
\begin{aligned}
\int_{0}^{u} & \Phi\left(\frac{a c \sqrt{y}}{u \sqrt{2}}\right) \frac{d}{y^{2}} \exp \left(-\frac{d}{y}\right) d y \\
= & \Phi\left(\frac{a c}{\sqrt{2 u}}\right) \exp \left(-\frac{d}{u}\right) \\
& -\int_{0}^{u} \exp \left(-\frac{d}{y}\right) \Phi^{\prime}\left(\frac{a c \sqrt{y}}{u \sqrt{2}}\right) \frac{a c}{2 u \sqrt{2 y}} d y \\
= & \Phi\left(\frac{a c}{\sqrt{2 u}}\right) \exp \left(-\frac{d}{u}\right) \\
& -\frac{a c}{4 u \sqrt{\pi}} \int_{0}^{u} \frac{1}{\sqrt{y}} \exp \left(-\frac{d}{y}-\frac{a^{2} c^{2} y}{4 u^{2}}\right) d y .
\end{aligned}
$$

Using integration method similar to that applied in the proof of Proposition 3, we can show

$$
\begin{gathered}
\frac{a c}{4 u \sqrt{\pi}} \int_{0}^{u} \frac{1}{\sqrt{y}} \exp \left(-\frac{d}{y}-\frac{a^{2} c^{2} y}{4 u^{2}}\right) d y \\
=-\frac{1}{2} \exp \left(\frac{a c \sqrt{d}}{u}\right)\left[1-\Phi\left(\frac{a c+2 \sqrt{d}}{\sqrt{2 u}}\right)\right] \\
+\frac{1}{2} \exp \left(-\frac{a c \sqrt{d}}{u}\right) \Phi\left(\frac{a c-2 \sqrt{d}}{\sqrt{2 u}}\right) .
\end{gathered}
$$

Hence,

$$
\begin{aligned}
(22)= & \Phi\left(\frac{a c}{\sqrt{2 u}}\right) \exp \left(-\frac{d}{u}\right)+\frac{1}{2} \exp \left(\frac{a c \sqrt{d}}{u}\right) \\
& {\left[1-\Phi\left(\frac{a c+2 \sqrt{d}}{\sqrt{2 u}}\right)\right] } \\
& -\frac{1}{2} \exp \left(-\frac{a c \sqrt{d}}{u}\right) \Phi\left(\frac{a c-2 \sqrt{d}}{\sqrt{2 u}}\right) .
\end{aligned}
$$

Substituting (24) for integral in (21), we get the desired result.

Proof of Result \#3.

$$
\begin{aligned}
P\left(Z_{t}\right. & \left.>u, Z_{t-1}>u\right) \\
= & 1-P\left(Z_{t} \leq u\right)-P\left(Z_{t-1} \leq u\right)+P\left(Z_{t} \leq u, Z_{t-1} \leq u\right) \\
= & 1-\exp \left(-\frac{d}{u}\right)-\exp \left(-\frac{d}{u}\right)+\exp \left(-\frac{c}{u}\right)\left\{2 \exp \left(-\frac{d}{u}\right)\right. \\
& -2 \exp \left(-\frac{d}{u}\right) \Phi\left(\frac{a c}{\sqrt{2 u}}\right) \\
& -\exp \left(\frac{a c \sqrt{d}}{u}\right)\left[1-\Phi\left(\frac{a c+2 \sqrt{d}}{\sqrt{2 u}}\right)\right] \\
& \left.+\exp \left(-\frac{a c \sqrt{d}}{u}\right) \Phi\left(\frac{a c-2 \sqrt{d}}{\sqrt{2 u}}\right)\right\} \text { by }(13), \\
= & \exp \left(-\frac{c}{u}\right)\left\{\exp \left(\frac{c}{u}\right)-2 \exp \left(-\frac{d-c}{u}\right)+2 \exp \left(-\frac{d}{u}\right)\right. \\
& -2 \exp \left(-\frac{d}{u}\right) \Phi\left(\frac{a c}{\sqrt{2 u}}\right) \\
& -\exp \left(\frac{a c \sqrt{d}}{u}\right)\left[1-\Phi\left(\frac{a c+2 \sqrt{d}}{\sqrt{2 u}}\right)\right] \\
(25)+ & \left.\exp \left(-\frac{a c \sqrt{d}}{u}\right) \Phi\left(\frac{a c-2 \sqrt{d}}{\sqrt{2 u}}\right)\right\} .
\end{aligned}
$$

Expanding (25) to the order $o\left(\frac{1}{u^{2}}\right)$, we get

$$
\begin{aligned}
(25)= & {\left[1-\frac{c}{u}+\frac{c^{2}}{2 u^{2}}+o\left(\frac{1}{u^{2}}\right)\right]\left\{1+\frac{c}{u}+\frac{c^{2}}{2 u^{2}}+o\left(\frac{1}{u^{2}}\right)\right.} \\
& -2\left[1-\frac{d-c}{u}+\frac{(d-c)^{2}}{2 u^{2}}+o\left(\frac{1}{u^{2}}\right)\right] \\
& +2\left[1-\frac{d}{u}+\frac{d^{2}}{2 u^{2}}+o\left(\frac{1}{u^{2}}\right)\right]-2\left[1-\frac{d}{u}+\frac{d^{2}}{2 u^{2}}\right. \\
& \left.+o\left(\frac{1}{u^{2}}\right)\right]\left[\frac{1}{2}+\frac{a c}{2 \sqrt{u \pi}}-\frac{a^{3} c^{3}}{24 u^{3 / 2} \sqrt{\pi}}+o\left(\frac{1}{u^{2}}\right)\right]
\end{aligned}
$$




$$
\begin{aligned}
&-\left[1+\frac{a c \sqrt{d}}{u}+\frac{a^{2} c^{2} d}{2 u^{2}}+o\left(\frac{1}{u^{2}}\right)\right]\left[\frac{1}{2}\right. \\
&\left.-\frac{a c+2 \sqrt{d}}{2 \sqrt{u \pi}}+\frac{(a c+2 \sqrt{d})^{3}}{24 u^{3 / 2} \sqrt{\pi}}+o\left(\frac{1}{u^{2}}\right)\right] \\
&+\left[1-\frac{a c \sqrt{d}}{u}+\frac{a^{2} c^{2} d}{2 u^{2}}+o\left(\frac{1}{u^{2}}\right)\right] \\
& {\left.\left[\frac{1}{2}+\frac{a c-2 \sqrt{d}}{2 \sqrt{u \pi}}-\frac{(a c-2 \sqrt{d})^{3}}{24 u^{3 / 2} \sqrt{\pi}}+o\left(\frac{1}{u^{2}}\right)\right]\right\} } \\
&= {\left[1-\frac{c}{u}+\frac{c^{2}}{2 u^{2}}+o\left(\frac{1}{u^{2}}\right)\right] } \\
& {\left[\frac{2 a c d}{u^{3 / 2} \sqrt{\pi}}-\frac{c^{2}-4 c d+d^{2}}{2 u^{2}}+o\left(\frac{1}{u^{2}}\right)\right] } \\
& \sim \frac{2 a c d}{\sqrt{\pi}} u^{-\frac{3}{2}}, \\
& \sim \operatorname{cst} P\left(Z_{t}>u\right)^{1 / \eta}, \text { because } P\left(Z_{t}>u\right) \sim d u^{-1} .
\end{aligned}
$$

Proof of Result \#4.

$$
\begin{aligned}
E\left(\exp \left\{-\frac{1}{Z_{t, \frac{1}{2}}}\right\}\right) & =\int_{0}^{\infty} \exp \left(-\frac{1}{z}\right) \exp \left(-\frac{d}{z}\right) \frac{d}{z^{2}} d z \\
& =\frac{d}{d+1} \int_{0}^{\infty} \exp \left(-\frac{d+1}{z}\right) \frac{d+1}{z^{2}} d z \\
& =\frac{d}{d+1} .
\end{aligned}
$$

Before calculating $E\left(\exp \left\{-\frac{1}{Z_{t, \frac{1}{2}}}-\frac{1}{Z_{t-1, \frac{1}{2}}}\right\}\right)$, we find the joint density $f(x, y)$ of $\left(Z_{t}, Z_{t-1}\right)$. Similar to $(21)$,

$$
\begin{aligned}
& P\left(Z_{t, \frac{1}{2}} \leq x, Z_{t-1, \frac{1}{2}} \leq y\right) \\
& \quad=2 \exp \left(-\frac{c}{x}\right) \exp \left(-\frac{d}{y}\right) \\
& \quad-2 \exp \left(-\frac{c}{x}\right) \int_{0}^{y} \Phi\left(\frac{a c \sqrt{w}}{x \sqrt{2}}\right) \frac{d}{w^{2}} \exp \left(-\frac{d}{w}\right) d w .
\end{aligned}
$$

Then

$$
\begin{aligned}
f(x, y)= & 2\left[\frac{c}{x^{2}} \exp \left(-\frac{c}{x}\right) \frac{d}{y^{2}} \exp \left(-\frac{d}{y}\right)\right. \\
& -\frac{c}{x^{2}} \exp \left(-\frac{c}{x}\right) \frac{d}{y^{2}} \exp \left(-\frac{d}{y}\right) \Phi\left(\frac{a c \sqrt{y}}{x \sqrt{2}}\right) \\
& \left.+\exp \left(-\frac{c}{x}\right) \frac{d}{y^{2}} \exp \left(-\frac{d}{y}\right) \Phi^{\prime}\left(\frac{a c \sqrt{y}}{x \sqrt{2}}\right) \frac{a c \sqrt{y}}{x^{2} \sqrt{2}}\right] \\
(26) \quad \equiv & 2\left[I_{1}-I_{2}+I_{3}\right] .
\end{aligned}
$$

Then

$$
\begin{aligned}
& E\left(\exp \left\{-\frac{1}{Z_{t, \frac{1}{2}}}-\frac{1}{Z_{t-1, \frac{1}{2}}}\right\}\right) \\
& \quad=2 \int_{0}^{\infty} \int_{0}^{\infty} \exp \left(-\frac{1}{x}-\frac{1}{y}\right)\left[I_{1}-I_{2}+I_{3}\right] d x d y
\end{aligned}
$$

and

$$
\begin{aligned}
& \int_{0}^{\infty} \int_{0}^{\infty} \exp \left(-\frac{1}{x}-\frac{1}{y}\right) I_{1} d x d y \\
& =\int_{0}^{\infty} \exp \left(-\frac{c+1}{x}\right) \frac{c}{x^{2}} d x \int_{0}^{\infty} \exp \left(-\frac{d+1}{y}\right) \frac{d}{y^{2}} d y \\
& \text { (28) }=\left(\frac{c}{c+1}\right)\left(\frac{d}{d+1}\right) \text {, } \\
& \int_{0}^{\infty} \int_{0}^{\infty} \exp \left(-\frac{1}{x}-\frac{1}{y}\right) I_{2} d x d y \\
& =\int_{0}^{\infty}\left\{\exp \left(-\frac{c+1}{x}\right) \frac{c}{x^{2}} \int_{0}^{\infty} \exp \left(-\frac{d+1}{d}\right) \frac{d}{y^{2}}\right. \\
& \left.\Phi\left(\frac{a c \sqrt{y}}{x \sqrt{2}}\right) d y\right\} d x \\
& =\int_{0}^{\infty}\left\{\exp \left(-\frac{c+1}{x}\right) \frac{c}{x^{2}} \frac{d}{d+1} \int_{0}^{\infty} \Phi\left(\frac{a c \sqrt{y}}{x \sqrt{2}}\right)\right. \\
& \left.d \exp \left(-\frac{d+1}{y}\right)\right\} d x \\
& =\int_{0}^{\infty}\left\{\operatorname { e x p } ( - \frac { c + 1 } { x } ) \frac { c } { x ^ { 2 } } \frac { d } { d + 1 } \left[1-\int_{0}^{\infty} \exp \left(-\frac{d+1}{y}\right)\right.\right. \\
& \left.\left.\Phi^{\prime}\left(\frac{a c \sqrt{y}}{x \sqrt{2}}\right) \frac{a c}{2 x \sqrt{2 y}} d y\right]\right\} d x \\
& =\left(\frac{c}{c+1}\right)\left(\frac{d}{d+1}\right)-\frac{d}{d+1} \int_{0}^{\infty} \int_{0}^{\infty} \exp \left(-\frac{c+1}{x}\right) \frac{c}{x^{2}} \\
& \exp \left(-\frac{d+1}{y}\right) \Phi^{\prime}\left(\frac{a c \sqrt{y}}{x \sqrt{2}}\right) \frac{a c}{2 x \sqrt{2 y}} d y d x \\
& =\left(\frac{c}{c+1}\right)\left(\frac{d}{d+1}\right)-\frac{d}{d+1} \int_{0}^{\infty} \exp \left(-\frac{c+1}{x}\right) \frac{c}{x^{2}} \frac{a c}{4 x \sqrt{\pi}} \\
& \int_{0}^{\infty} \frac{1}{\sqrt{y}} \exp \left(-\frac{d+1}{y}-\frac{a^{2} c^{2} y}{4 x^{2}}\right) d y d x \\
& =\left(\frac{c}{c+1}\right)\left(\frac{d}{d+1}\right)-\frac{d}{d+1} \int_{0}^{\infty} \exp \left(-\frac{c+1}{x}\right) \frac{c}{x^{2}} \frac{1}{2} \\
& \exp \left(-\frac{a c \sqrt{d+1}}{x}\right) d x \\
& =\left(\frac{c}{c+1}\right)\left(\frac{d}{d+1}\right)-\frac{1}{2}\left(\frac{d}{d+1}\right) \\
& \int_{0}^{\infty} \exp \left(-\frac{c+1+a c \sqrt{d+1}}{x}\right) \frac{c}{x^{2}} d x \\
& \text { (29) }=\left(\frac{c}{c+1}\right)\left(\frac{d}{d+1}\right)-\frac{1}{2}\left(\frac{d}{d+1}\right)\left(\frac{c}{c+1+a c \sqrt{d+1}}\right) \text {, }
\end{aligned}
$$

where the sixth equality is obtained by a calculation similar to that applied in obtaining equation (23).

$$
\begin{aligned}
\int_{0}^{\infty} & \int_{0}^{\infty} \exp \left(-\frac{1}{x}-\frac{1}{y}\right) I_{3} d x d y \\
& =\int_{0}^{\infty} \int_{0}^{\infty} \exp \left(-\frac{c+1}{x}\right) \exp \left(-\frac{d+1}{y}\right) \frac{d}{y^{3 / 2}} \frac{a c}{2 x^{2} \sqrt{\pi}}
\end{aligned}
$$

An extension of max autoregressive models 265 


$$
\begin{aligned}
& \exp \left(-\frac{a^{2} c^{2} y}{4 x^{2}}\right) d x d y \\
= & \int_{0}^{\infty} \exp \left(-\frac{c+1}{x}\right) \frac{a c d}{2 x^{2} \sqrt{\pi}} \int_{0}^{\infty} \frac{1}{y^{3 / 2}} \exp \left(-\frac{d+1}{y}\right. \\
& \left.-\frac{a^{2} c^{2} y}{4 x^{2}}\right) d y d x \\
= & \int_{0}^{\infty} \exp \left(-\frac{c+1}{x}\right) \frac{a c d}{2 x^{2}} \frac{1}{\sqrt{d+1}} \exp \left(-\frac{a c \sqrt{d+1}}{x}\right) d x \\
= & \frac{1}{2}\left(\frac{a c d}{\sqrt{d+1}}\right) \int_{0}^{\infty} \frac{1}{x^{2}} \exp \left(-\frac{c+1}{a c d}\right) d x \\
= & \frac{1}{2} \frac{a c \sqrt{d+1}}{\sqrt{d+1}(c+1+a c \sqrt{d+1})},
\end{aligned}
$$

where the third equality is obtained by a calculation similar to that applied in the proof of Proposition 3.

Combining the results of (27), (28), (29), (30), we get

$$
\begin{aligned}
E( & \left.\exp \left\{-\frac{1}{Z_{t, \frac{1}{2}}}-\frac{1}{Z_{t-1, \frac{1}{2}}}\right\}\right) \\
= & 2\left[\left(\frac{c}{c+1}\right)\left(\frac{d}{d+1}\right)-\left(\frac{c}{c+1}\right)\left(\frac{d}{d+1}\right)\right. \\
& +\frac{1}{2}\left(\frac{d}{d+1}\right)\left(\frac{c}{c+1+a c \sqrt{d+1}}\right) \\
& \left.+\frac{1}{2} \frac{a c d}{\sqrt{d+1}(c+1+a c \sqrt{d+1})}\right] \\
= & \frac{c d+a c d \sqrt{d+1}}{(d+1)(c+1+a c \sqrt{d+1})} .
\end{aligned}
$$

Received 28 May 2011

\section{REFERENCES}

[1] Cooley, D., Davis, R. A., and Naveau, P. (2007). Prediction for max-stable processes via an approximated conditional density. Technical Report. Department of Statistics, Colorado State University.

[2] Crowder, M. J. (1989). A multivariate distribution with Weibull components. J. Roy. Statist. Soc. Ser. B, 51, 93-108. MR0984996

[3] Davis, R. A. and Resnick, S. I. (1989). Basic properties and prediction of Max-ARMA processes. Adv. Appl. Prob., 21, 781803. MR1039628

[4] Deheuvels, P. (1983). Point processes and multivariate extreme values. Jour. of Multi. Anal. 13, 257-272. MR0705550

[5] Fougéres A.-L., Nolan, J. P., and Rootzén, H. (2009). Models for dependent extremes using stable mixtures. Scandinavian Journal of Statistics, 36, 42-59. MR2508330
[6] Hall, P., Peng, L. and Yao, Q. (2002). Moving-maximum models for extrema of time series. Journal of Statistical Planning and Inference, 103, 51-63. MR1896983

[7] Hougandd, P. (1986). A class of multivariate failure time distributions. Biometrika, 73, 671-678. MR0897858

[8] Ledford, A. W. and TAwn, J. A. (1996). Statistics for near independence in multivariate extreme values. Biometrika, 55, 169187. MR1399163

[9] Ledford, A. W. and Tawn, J. A. (1997). Modelling dependence within joint tail regions, Journal of the Royal Statistical Society, Series B, 59, 475-499. MR1440592

[10] Sibuya, M. (1960). Bivariate extreme statistics, I. Ann. Inst. Statist. Math, 11, 195-210. MR0115241

[11] Smith, R. L., and Weissman, I. (1996). Characterization and estimation of the multivariate extremal index. Unpublished manuscript, University of North Carolina. http://www.stat.unc.edu/postscript/rs/extremal.pdf.

[12] TANG, R., ShaO, J., and Zhang, Z. (2010). Sparse moving maxima models for extreme dependence in multivariate financial time series. Manuscript.

[13] TAwn, J. A. (1990). Modelling multivariate extreme value distributions. Biometrika, 77, 245-253.

[14] Tong, H. and Lim, K. S. (1980). Threshold autoregression, limit cycles and cyclical data (with discussion), J. Roy. Statist. Soc. Ser. B, 42, 245-292.

[15] Toulemonde, G., Guillou, A., Naveau, P., Vrac, M., and Chevallier, F. (2010). Autoregressive models for maxima and their applications to $\mathrm{CH}_{4}$ and $\mathrm{N}_{2} \mathrm{O}$, Environmetrics, 21, 189-207.

[16] Zhang, Z. and Sмiтh, R. L. (2004). The behavior of multivariate maxima of moving maxima processes. Journal of Applied Probability, 41, 1113-1123. MR2122805

\section{Philippe Naveau}

Laboratoire des Sciences du Climat et de I'Environnement

LSCE-IPSL-CNRS

91191 Gif-sur-Yvette

France

E-mail address: philnova21@gmail.com

\section{Zhengjun Zhang}

Department of Statistics

University of Wisconsin

Madison, WI 53706

USA

E-mail address: zjz@stat.wisc.edu

Bin Zhu

Department of Statistics

University of Wisconsin

Madison, WI 53706

USA

E-mail address: binzhu@stat.wisc.edu 